Dieses Dokument ist eine Zweitveröffentlichung (Postprint) /

This is a self-archiving document (postprint):

Mandy Korzetz, Romina Kühn, Uwe Aßmann, Thomas Schlegel

Investigating Mobile Device-Based Interaction Techniques for

Collocated Merging

Erstveröffentlichung in / First published in:

HCII: International Conference on Human-Computer Interaction, Copenhagen 2020. Cham: Springer, S. 92 -108. ISBN 978-3-030-50513-4

DOI: https://doi.org/10.1007/978-3-030-50513-4_7

Diese Version ist verfügbar / This version is available on:

https://nbn-resolving.org/urn:nbn:de:bsz:14-qucosa2-755073 


\title{
Investigating Mobile Device-Based Interaction Techniques for Collocated Merging
}

\author{
Romina Kühn ${ }^{1}$, Mandy Korzetz ${ }^{1}$, Felix Kallenbach ${ }^{1}$, Karl Kegel ${ }^{1}$, \\ Uwe Aßmann $^{1}$, and Thomas Schlegel ${ }^{2}$ \\ 1 TU Dresden, Institute of Software and Multimedia Technology, Dresden, Germany \\ \{romina.kuehn, mandy.korzetz, uwe.assmann\}@tu-dresden.de \\ 2 Karlsruhe University of Applied Science, Institute for Ubiquitous Mobility Systems \\ Karlsruhe, Germany \\ thomas.schlegel@hs-karlsruhe.de
}

\begin{abstract}
In mixed-focus collaboration, group members create content both individually as a kind of groundwork for discussion and further processing as well as directly together in group work sessions. In case of individual creation, separate documents and contents need to be merged to receive an overall solution. In our work, we focus on mixed-focus collaboration using mobile devices, especially smartphones, to create and merge content. Instead of using emails or messenger services to share content within a group, we describe three different mobile device-based interaction techniques for merging that use built-in sensors to enable ad-hoc collaboration and that are easy and eyes-free to perform. We conducted a user study to investigate these merging interactions. Overall, 21 participants tested the interactions and evaluated task load and User Experience (UX) of the proposed device-based interactions. Furthermore, they compared the interactions with a common way to share content, namely writing an email to send attached content. Participants gave valuable user feedback and stated that our merging interaction techniques were much easier to perform. Furthermore, we found that they were much faster, less demanding, and had a greater UX than email.
\end{abstract}

Keywords: Collocated Interaction $\cdot$ Device-Based Interaction $\cdot$ Merging · Mixed-Focus Collaboration · Mobile Phone.

\section{Introduction}

In collocated mixed-focus collaboration, as proposed by Gutwin and Saulberg [6], group members move back and forth between individual and shared tasks to reach a common goal in terms of fulfilling their collaborative task. While working individually, group members typically perform collaboration activities [13] such as creating or editing content as partial solution of an overall collaborative solution. Then, after the individual tasks are completed the results need to be shared completely or merged in parts for further processing within the group. 


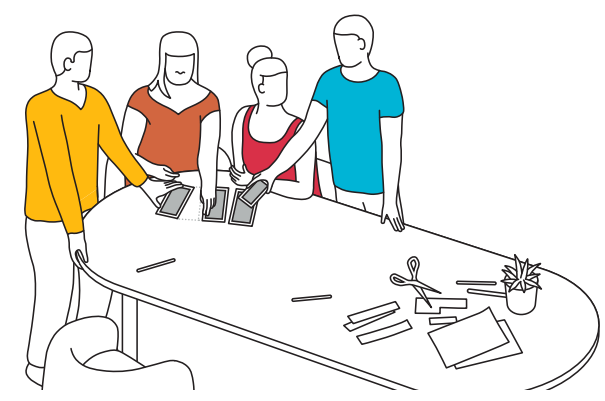

Fig. 1. Collocated collaboration scenario with merging interactions. Group members apply our proposed interaction techniques with their mobile devices to merge content.

A common way to share content across mobile phones is to send an email with attached documents or using a messaging service, e. g. WhatsApp ${ }^{3}$. For more enhanced sharing, people also use cloud services such as Dropbox ${ }^{4}$ or similar tools that work as file hosting services. However, there are different issues with using these techniques, e. g. limited storage space or higher complexity of selecting files when switching between several applications. Switching leads to media breaks, which can be awkward. There are several ways and tools that enable group members to work simultaneously, which would eliminate the need of sharing files explicitly. For example, Google Docs ${ }^{5}$ facilitates collaborative text writing and $\mathrm{Git}^{6}$ can be used for collaborative software development. However, these tools do not support switching between individual and collaborative work and the resulting communicative merging between group members in terms of face-to-face communication, which would be beneficial for collocated collaboration [4]. We focus on mobile devices especially smartphones as ubiquitous devices $[1,2]$ and their usage in collocated mixed-focus collaboration. As stated by Lucero et al. [17] and Dong et al. [5] the main issue with applying smartphones in collaboration is a lack of intuitive and easy-to-perform interactions. To address this issue, we utilize mobile device-based interaction techniques that were presented by Korzetz et al. [11]. Such interactions aim at facilitating unobtrusive interactions in collocated multi-user tasks and enabling quick access to various device functions. Furthermore, they focus on their spatial and tangible behavior by using the built-in sensors instead of the display's representations and, therefore, do not need much user attention. By applying mobile device-based interactions, we aim at integrating mobile devices in collocated collaborative merging in an easy and intuitive way without affecting communication adversely. We investigate three specific mobile device-based interaction techniques for merging results in collocated mixed-focus collaboration (see Fig. 1).

\footnotetext{
3 https://www.whatsapp.com/

${ }^{4}$ https://www.dropbox.com/

${ }^{5}$ https://docs.google.com

${ }^{6}$ https://git-scm.com/
} 
To present the results of our investigations, we structured the paper as follows: First, we give an overview on related work that describes approaches on merging and sharing content with mobile devices. Then we describe our proposed interaction techniques that aim at merging content more easily compared to common merging strategies. This aim was investigated in a user study we performed by means of an interactive prototype. We present the results and conclude with recommendations and future work.

\section{Related Work}

Using mobile devices as physical interfaces enables users to interact efficiently in certain situations and to provide fast and easy-to-use input possibilities [15, 23]. Korzetz et al. [11] proposed the mobile spaces model (MSM) to facilitate the design of such mobile device-based interaction techniques to support individual as well as collaborative usage scenarios. As device-based interaction techniques are lightweight and additionally have been investigated and assessed as effective possibility to support collaboration activities $[13,18]$, we utilize the concepts of the MSM for our merging interactions. Exchanging and sharing information and media with other devices is very important in general, e. g. for creating joint results in collaboration. In this context, we identified two main areas of related work that have influenced the development of our merging interactions, namely (1) sharing interactions with a mobile device in general and (2) sharing interactions between two or more mobile devices.

MobiSurf [24] integrates various personal mobile devices and one shared interactive surface in a home setting to support solving collocated collaborative tasks. Content that should be discussed, e. g a web page, is transferred by touching the interactive surface with the mobile device, then the browser of the interactive surface loads the page. Ubi-Jector [16] is a system that provides a shared information screen utilizing the users' personal mobile devices in a casual meeting environment. The system provides a visual interface to provide file access to the attendees automatically when connecting to the system. To move information between a mobile device and a large display, several interaction techniques were proposed. The idea of cross-device sharing can be traced back to the work of Rekimoto [22] who proposed the concept of pick-and-drop to use direct manipulation techniques to transfer data between displays using a pen. Shoot-and-copy is an interaction technique to transfer information from public displays to mobile phones by using the built-in camera [3]. The user captures an arbitrary region of the large display and then the corresponding data is sent to the users' device. The interaction techniques pinch, swipe, throw and tilt were investigated to move information vice versa to a large display, e. g. by Paay et al. [21]. Although these techniques were rated as effective and easy-to-learn device-based interactions, they do not support ad-hoc collaboration because an additional and mostly bigger device is required. Our merging interactions are designed to support spontaneous collaboration by solely utilizing mobile devices. 
Some research already investigates interaction techniques for sharing between multiple mobile devices. Whereas Ohta [19] proposed a pinch gesture across the borders of two mobile devices to establish a network connection between them and thereby enable moving a photograph over the device boundaries, Hinckley explored bumping devices [7] and stitching gestures [8] for tiling together displays. These approaches use devices as physical interface but concentrate on establishing a device connection and providing a joint viewing area instead of sharing information among a group. However, our interaction approach aims at sharing and especially merging parts of content to enable further processing.

\section{Merging Interaction Techniques}

To merge individually created content there are several aspects and challenges to address. First, the type of data and media can influence the way of merging. Content can include text, several kinds of illustrations, e. g. images, photos, and diagrams, or videos as well as combinations of different kinds of media. Whereas text can be merged in small units, e. g. sentences or even phrases, visuals are usually merged either completely or not at all. This aspect mainly influences the selection of content that needs to be merged, e. g. a number of images or concrete text phrases, but also the merging itself, e. g. the location within a given text where the phrases need to be added. Depending on the way of working individually in mixed-focus collaboration, single tasks can be performed as alternatives or subtasks to complete a group work more efficiently. Hence, solutions or partial solutions can overlap in different ways and need to be merged before they are further edited. For example, five members of a student group have to prepare a mind map with three subtopics. Whereas one member can work on one subtopic, two members work on the second subtopic and two other members on the third subtopic. Thus, they create alternatives of the subtopics. Alternatives often need to be discussed first within the subgroup before they can be further edited by the whole group. This leads to the need to share content within subgroups bidirectionally so that each subgroup member has a joint solution. Consequently, the direction is also important for merging. Finally, the user roles in collaboration can affect the way of merging content. In case, there is one concrete person using one specific mobile device that collects all parts of an overall solution, content is merged on that specific device. This can be useful for editing together on one device before sharing within the whole group. In the other case, merged content can be provided for each group member immediately. Figure 2 illustrates these aspects for the merging activity.

For our proposed interaction techniques, we focus on the direction of merging content, namely bidirectional and unidirectional merging. We also add the option that content is not merged at all and instead excluded from the merging process. We further refer to the way of merging that is derived from the direction as merging style. In case (partial) solutions do not overlap they can be united easily by adding one solution to another solution and vice versa (bidirectional merging). Composing is necessary in case of partial overlapping as content can 


\section{Overlap of Partial Solutions}

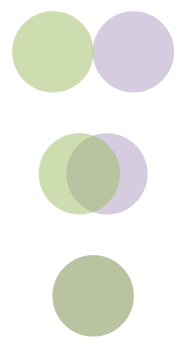

\section{Merging \\ Direction}

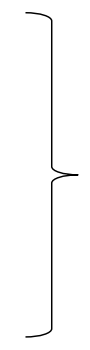

bidirectional

unidirectional

none

\section{Merging \\ Style}

Unite

Compose

Exclude

\section{Interaction \\ Technique}

Tape to Unite

(Tape)

Pour to Compose

(Pour)

Turn to Exclude

(Turn)

Fig. 2. Overview of relevant merging aspects.

be complemented partially in one direction from one device to another device. Content of several devices that overlaps completely can be excluded. The proposed merging interactions were introduced by Korzetz et al. [9] and are shown in Figure 3. They are inspired by everyday actions and address the mixed-focus collaboration activity sharing results by setting in after group members compared several interim results and want to generate an overall solution [13]. They are designed to be easy and eyes-free to perform without further equipment. Furthermore, they address the concepts of the mobile spaces model (MSM) [11].

Tape to Unite (Fig. 3a) enables users to directly add a part to an overall solution, e. g. one part of a bigger mind map or a text section of a chapter. The interaction is performed as follows: First, the devices are placed next to each other. Second, the user performs a swipe gesture on the screens of the involved devices simultaneously (see Fig. 3a). Depending on the position where the devices are placed next to each other, the content is merged. For example, text can be added at the end of a continuous text by placing devices on the short sides of the smartphones and performing the tape gesture along those sides. While the devices "stick together" the content can be presented on each involved device to enlarge the presentation area as described, e. g. by Ohta and Tanaka [20]. When the devices are detached a united solution remains on both devices. We further refer to this interaction technique as Tape.

The Pour to Compose (or Pour) interaction is more versatile in terms of the merging options. This interaction can be used in case users want to put together several pieces for an overall solution. For example, to create a joint text several text blocks of different group members can be merged with this interaction technique on one or several devices. Pour is performed by pouring the content from one device out into another device as shown in Figure 3b. The sending device (left) is tilted above the receiving device (right) and the content flows into the receiving device. Varying the tilt angle increases or decreases the speed of transferring the data. For selecting the content of the sending device and specifying the position to merge the content on the receiving device, additional 


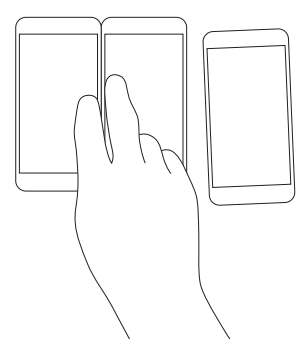

(a) Tape to Unite

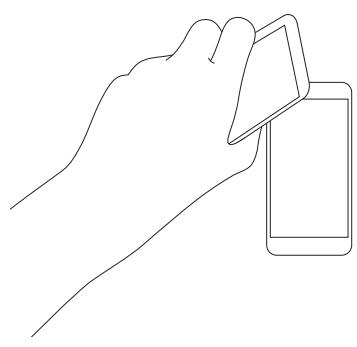

(b) Pour to Compose

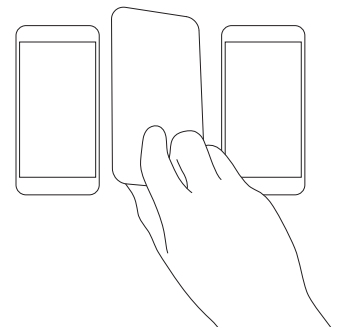

(c) Turn to Exclude

Fig. 3. Device-based interactions for collocated merging

pick and point gestures can be used. For example, a touch-based lasso gesture can be used to pick content and a finger tap can be used for pointing. In contrast to the Tape interaction, Pour is unidirectional, which means that the pouring device only receives content when it changes roles and becomes a receiving device.

The Turn to Exclude (or Turn) interaction (see Fig. 3c) enables users to exclude a solution from further discussions by turning the device [12]. This interaction technique focuses on the digital content of the other devices and excludes content successively if necessary. Excluding can be useful in case a proposed partial solution is not suitable for further processing.

\section{User Study}

We conducted a user study to examine the proposed interaction techniques concerning their task load and UX using the standardized NASA Task Load Index ${ }^{7}$ (NASA-TLX) and the User Experience Questionnaire ${ }^{8}$ (UEQ). Furthermore, we compared the proposed merging interactions with a commonly used technique for sharing content, namely attaching files to an email and sending it to another device. In the following section, we describe the setup and design of our user study, participants, and our interactive prototype in detail.

\subsection{Participants}

We recruited 21 unpaid participants ( 5 female) from age 24 to $42(\mathrm{M}=32.2$, $\mathrm{SD}=5.3)$ via email or personally. Participants assessed their smartphone experience on a 5-point Likert scale ranging from 1 (little experience) to 5 (expert). Participants had an overall high prior experience in using smartphones $(\mathrm{M}=4.2$, $\mathrm{SD}=1.0)$. They stated that they mainly use their smartphones for messaging and communication services (20), browsing the internet (14), and entertainment

\footnotetext{
7 https://humansystems.arc.nasa.gov/groups/TLX/

8 https://www.ueq-online.org/
} 

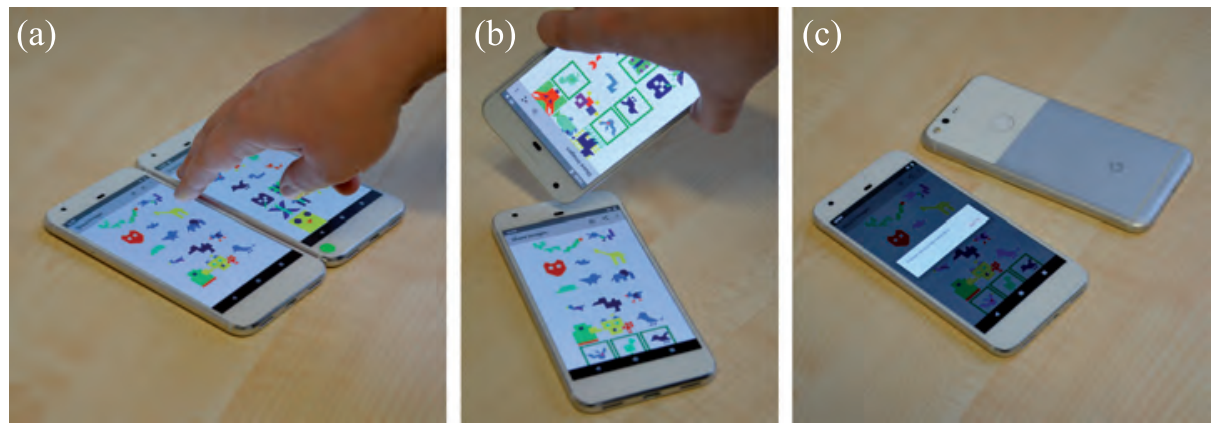

Fig. 4. Interactive prototype. (a) Tape interaction to unite content. (b) Pour for composing content. (c) Turn to exclude content.

purposes (12), e. g. watching videos or gaming. The majority of participants (16) use smartphones with Android as operating system. Furthermore, they stated that they mainly use messaging services (15), cloud services (12), and email (6) for merging content depending on their individual goal.

\subsection{Interactive Prototype}

We implemented the above-mentioned interaction techniques using the MilkyWay toolbox [10], a toolbox to facilitate fast and easy development of mobile device-based interactions. The toolbox provides a simple connection establishment between devices using Wi-Fi direct and a gesture editor that combines several movements and gestures to create one joint gesture set. To conduct each interaction properly all devices that were used for the user study had to provide an accelerometer, a gyroscope, and a proximity sensor as well as a touch display. The participants used two Google Pixel smartphones with Android version 10.0, which we provided for the user study to guarantee the availability of the necessary sensors and to control the setting. The Tape interaction used the touch screen and recognized the touch gesture within a predefined area. For Pour the sending device recognized the changing orientation using the accelerometer. Additionally, the receiving device recognized the other device using the proximity sensor. Finally, for Turn the gyroscope and the proximity sensor detected the turning of the device. As soon as a merging interaction was recognized the respective merging style was triggered.

Our interactive prototype was built as an image sharing application (see Fig. 4). We provided several simple pixel graphics for merging into one gallery for further processing, e. g. starting a joint exhibition in an art class. The application presented the provided images. The action bar contained a button to select all images at once, a sharing button for sending pictures via email, and a shortcut for resetting the gallery. Within the gallery, images could be selected or deselected by touching the respective image. Each merging interaction technique was implemented as described in section 3 . We merged images by adding them to the 
end of the gallery. As soon as users performed an interaction, they received visual feedback either as added images or textual information. Furthermore, the device vibrated in order to give haptic feedback. To compare the interaction techniques, we implemented a common alternative way to share content. A sharing button enabled the user to open the Gmail application and to attach the selected images to an email for sending it. The other device received the email, downloaded the image manually, and added it to the gallery automatically. We decided to use email as alternative because it is (still) a very common way to share data between several devices, data is not compressed automatically as it is the case when using messaging services, and it is also used by people who do not use cloud services.

\subsection{Procedure}

To investigate the interaction techniques accordingly, participants performed the user study as follows. It started with an explanation of the global procedure and the goal of the user study. The participants were asked to describe their usual way of merging content. Then, the study leader presented the three merging interactions by demonstration and described the email interaction as well as the study application itself. The participants were asked to merge either all images, several selected images, or no images with our proposed interaction techniques and via email. After each performed interaction technique, participants completed the NASA-TLX questionnaire as well as the UEQ. Afterwards, the study leader reset the gallery for the next merging task. This procedure was repeated with the other interactions. Overall, the participants were asked to perform each merging style task (Unite, Compose, and Exclude twice using email and our merging interactions. To address the order bias, we permuted the order of interactions. A session concluded with an interview concerning final comments about the interactions and their potential usage in collaborative scenarios as well as a short questionnaire to collect demographic data. During a session the study leader took additional notes regarding a participant's performance and remarks. A session took about 35 minutes $(\mathrm{M}=35.2, \mathrm{SD}=6.6)$.

\subsection{Design}

We realized a within-subject design with the merging style as independent variable. We had two conditions: common email merging and using our device-based merging interactions Tape, Pour, and Turn. The dependent variable was the task completion time. To avoid learning effects, we counterbalanced the testing order of all interactions.

\section{Results}

We examined the task load as well as the UX of each interaction technique using the NASA-TLX questionnaire respectively the User Experience Questionnaire 


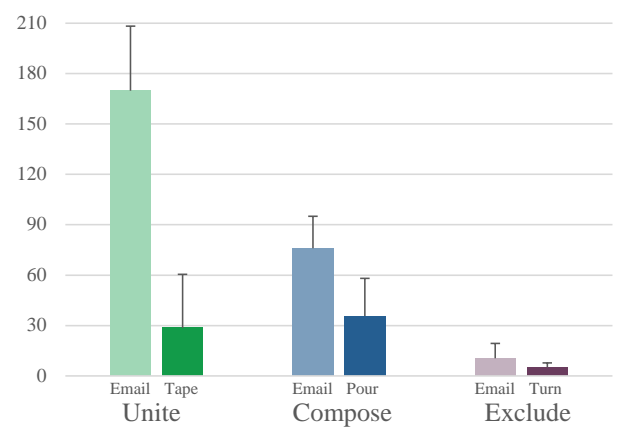

(a) Task Completion Time in Seconds

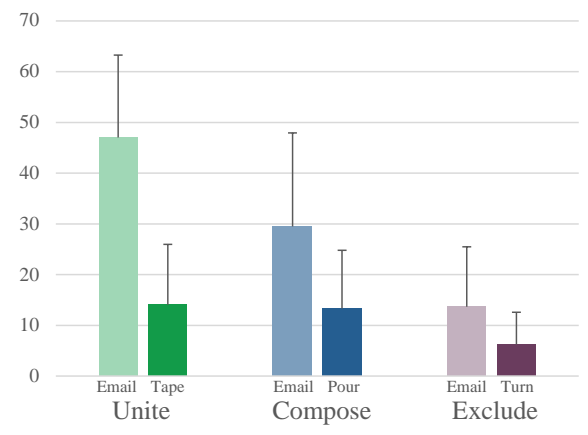

(b) Task Load

Fig. 5. Means and standard deviations of (a) task completion time in seconds and (b) task load per merging style and condition.

(UEQ) [14]. The NASA-TLX assesses task load on a scale from 1 (least demanding) to 100 (very demanding). The UEQ includes three main scales: attractiveness, pragmatic quality (measuring perspicuity, efficiency, and dependability) and hedonic quality (measuring stimulation and novelty). These three dimensions help us to evaluate UX aspects. User Experience was assessed on a 7-point Likert scale. In the following section, we describe the results of our user study.

\subsection{Task Completion Time}

We compared the task completion time per merging style using either email or our merging interactions. Fig. 5a illustrates the results from the task completion times by displaying the mean values (M) and standard deviations (SD). Measuring the task completion time started after we described the particular task and stopped after participants received the feedback of a successful merging. Uniting content on both devices took the participants between 90 and 222 seconds $(\mathrm{M}=169.8, \mathrm{SD}=38.5)$ using email and between 6 and 157 seconds $(\mathrm{M}=28.5$, $\mathrm{SD}=32.0$ ) applying the Tape interaction. Whereas for sending emails the standard deviation is relatively low, Tape performed one time much worse in contrast to the other sessions because of technical issues. Eliminating the session with a task completion time of 157 seconds, using Tape took between 6 and 53 seconds $(\mathrm{M}=22.1, \mathrm{SD}=12.8)$, which shows better results.

Composing content on one device took between 50 and 118 seconds $(\mathrm{M}=76.0$, $\mathrm{SD}=19.0)$ using email and between 12 and 86 seconds $(\mathrm{M}=35.7, \mathrm{SD}=22.4)$ applying the Pour interaction both including the selection of the content first. For excluding the content, participants took between 2 and 38 seconds $(\mathrm{M}=10.5$, $\mathrm{SD}=8.9)$ using email and between 2 and 11 seconds $(\mathrm{M}=5.2, \mathrm{SD}=2.6)$ applying the Turn interaction. Using email for excluding ranged from doing nothing to writing a short text via email that the participant won't send any images. We performed an analysis of variance (ANOVA) to investigate effects of the merging 


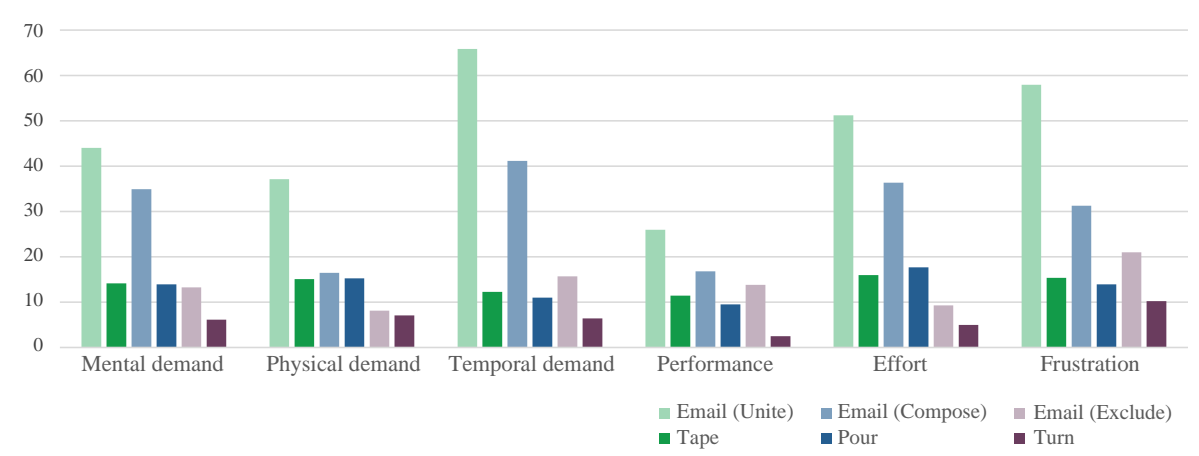

Fig. 6. Comparison of TLX means per criteria for all interactions after usage.

style conditions on task completion time. The analysis shows that for all merging styles the results are statistical significant: Unite $\left(F_{1,20}=233.298, p<.0001\right)$, Compose $\left(F_{1,20}=89.657, p<.0001\right)$, and Exclude $\left(F_{1,20}=9.355, p<.01\right)$. The results show a significantly longer task completion time for sending emails than using our proposed merging interactions. Although, the participants were all used to send images via email, the process of selecting images, attaching them to an email, waiting for the email to arrive at the other device, downloading the images and pasting them into the gallery was quite cumbersome and more complex in contrast to the merging interactions, especially for uniting content. There are less differences in the task completion times between email and Pour due to the lower complexity of the task. Overall, the standard deviation values of the task completion time were relatively high for the merging interactions because the interactions were all new to the participants and were performed for the first time during the user study. Consequently, some participants had to try the merging interactions several times. However, the merging interactions still performed significantly better than sending an email.

\subsection{Comparison of Task Load}

After completing each task using either email or a merging interaction, participants were asked to fill out the NASA-TLX to assess the perceived individual task load. Fig. 6 presents a comparison of the TLX means of all criteria for all interactions. We used ANOVA to investigate the statistical significance for the overall task load of each interaction. The results show that the effect of the interaction (email vs. merging interaction) on the task load was statistically significant for all merging styles: Unite $\left(F_{1,20}=52.880, p<.0001\right)$, Compose $\left(F_{1,20}=36.994, p<.0001\right)$, and Exclude $\left(F_{1,20}=7.500, p<.05\right)$. However, as shown in Fig. 6 the differences for the merging style Exclude are lower than for the other merging styles. As shown in Fig. 5b the overall task load for sending emails was much higher than for the respective merging interaction. For Unite, we found very high temporal demand due to the complexity of sending an email 


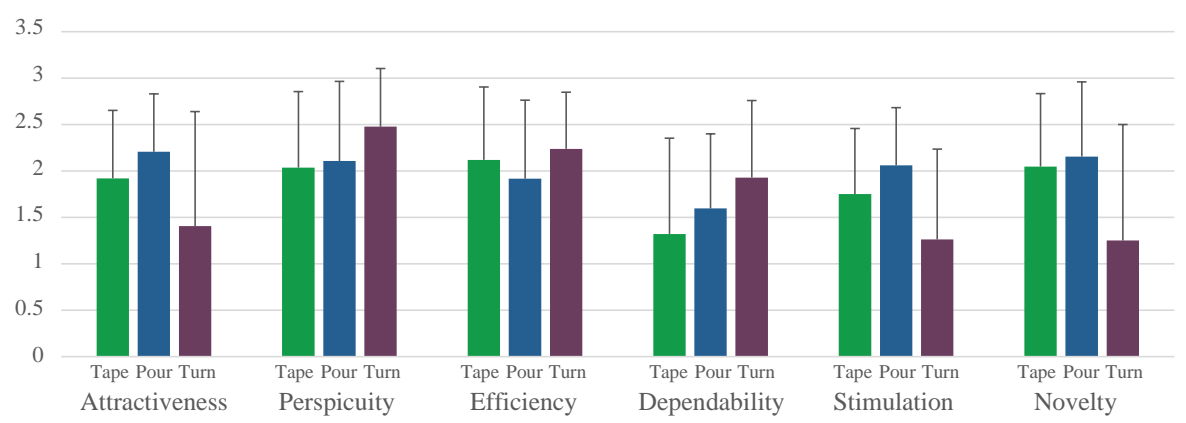

Fig. 7. UX ratings (means and standard deviation) of merging interaction techniques.

in both directions, which correlates with the measured task completion time and which we assume also led to high frustration. We found a similar valuation for sending emails for Compose where especially temporal demand and effort are high. The proposed merging interactions perform better for every single criteria.

\subsection{User Experience of Merging Interaction Techniques}

Additionally to the task load evaluation, we asked the participants to fill out the standardized User Experience Questionnaire (UEQ). The UEQ includes 26 word pairs that can characterize a product. These pairs are assessed by the participants on a 7-point Likert scale and are mapped to attractiveness, perspicuity, efficiency, dependability, stimulation, and novelty. Fig. 7 shows the means and standard deviations of the merging interaction techniques Tape, Pour, and Turn in terms of the UX ratings. Values $>0.8$ represent a positive rating, which shows an overall positive evaluation for all three merging interaction techniques. However, we wanted to have a closer look at the particular ratings.

Whereas for Tape, the single values of perspicuity, efficiency, and novelty culminate in relatively high means, the mean value of dependability is significantly lower with a high standard deviation $(\mathrm{SD}=1.03)$. From that, we derive that performing the interaction did not work flawlessly all the time. This correlates with our observation that some participants had to perform the Tape gesture several times before it was recognized. However, in general Tape was evaluated positively with a high attractiveness, pragmatic, and hedonic quality. For Pour, especially attractiveness and hedonic factors (stimulation and novelty) were rated high with lower standard deviations. Some participants also mentioned that they enjoyed performing the interaction very much. However, pragmatic quality aspects, especially efficiency and dependability, need to be improved to guarantee a high usability. We observed that some participants also had to perform the Pour interaction several times and in different ways. We assume that this led to devaluation. In contrast, the Turn interaction was rated best concerning pragmatic quality aspects which we interpret as Turn is a very explicit and comprehensible interaction technique. However, aspects in terms 
Table 1. Results from the t-test with an alpha level $\alpha=0.01$ comparing merging interaction technique and email.

\begin{tabular}{|l|l|l|l|}
\hline UX aspect & Unite & Compose & Exclude \\
\hline Attractiveness & $p<.0001$ & $p<.0001$ & $p<.01$ \\
Perspicuity & $p<.0001$ & $p<.0001$ & $p<.01$ \\
Efficiency & $p<.0001$ & $p<.0001$ & $p<.01$ \\
Dependability & $p<.01$ & $p>.01$ & $p>.01$ \\
Stimulation & $p<.0001$ & $p<.0001$ & $p<.0001$ \\
Novelty & $p<.0001$ & $p<.0001$ & $p<.0001$ \\
\hline
\end{tabular}

of attractiveness and hedonic quality were rated worse with high values of the standard deviation in contrast to Tape and Pour. We trace these ratings to a very easy and short interaction which is already known from muting an incoming call $^{9}$ and consequently not new to some participants. Overall, from the UEQ we can derive that our merging interaction techniques were rated positively.

\subsection{Comparison of User Experience}

To assess the results from the UEQ better, we compared the participants' UX evaluation of the merging interactions with one common alternative for sharing content: sending an email with attached files. The results show very strong differences in rating the various UX aspects of the merging interactions compared to sending an email. Fig. 8 illustrates the means and standard deviations of all interactions. Beside the partially much worse rating for email concerning attractiveness and hedonic quality in general, which we explain with the fact that sending emails is a very common concept, email also performed worse for pragmatic quality. Especially for Tape, the email condition performed much worse concerning perspicuity and efficiency. This rating correlates with the overall TLX rating of the Unite email interaction. Especially, the TLX showed higher temporal demands, effort, and frustration comparing email and Tape. For Pour, our analysis of the UX questionnaire showed a similar but mitigated effect in terms of pragmatic quality. We assume that the higher temporal demand and the more process steps for selecting the images, sending an email, and downloading the images led to devalued efficiency. This observation would also explain, why Exclude was rated more similar in both conditions.

We performed a t-test with an alpha level of $\alpha=0.01$ to check if the scale means differ significantly. Table 1 shows the results in detail. For Unite, the means for all scales differ significantly. For Compose as well as Exclude we also found significant differences for nearly all scales except dependability with $p=.0303$ for Compose and $p=.0194$ for Exclude.

\footnotetext{
${ }^{9}$ https://www.samsung.com/ca/support/mobile-devices/what-are-the-advancedfeatures-available-on-my-galaxy-note8/
} 


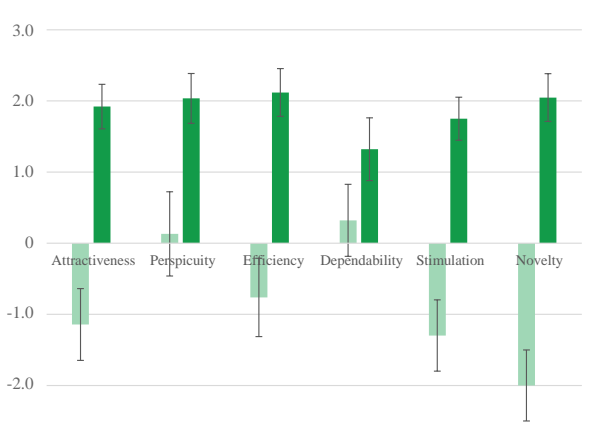

(a) Comparison of Tape and Email

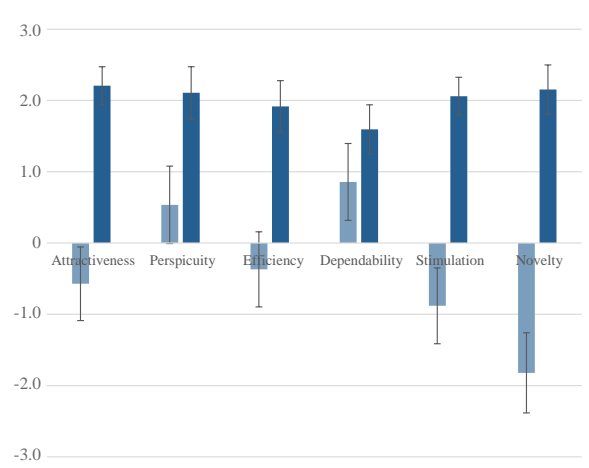

(b) Comparison of Pour and Email

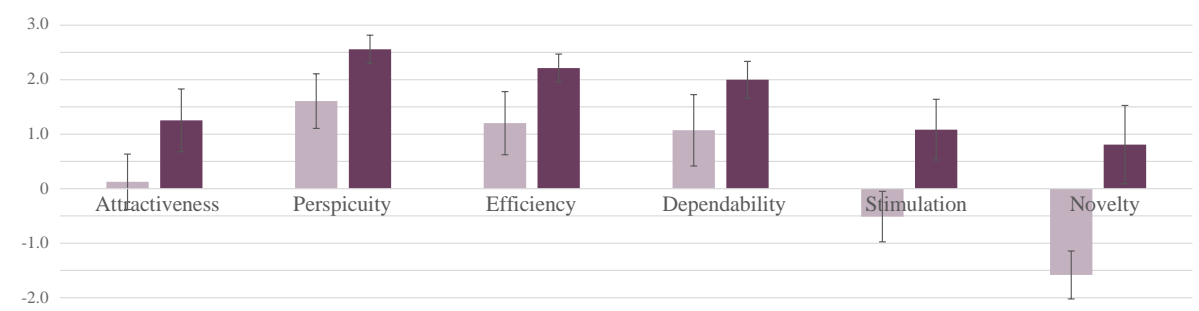

(c) Comparison of Turn and Email

Fig. 8. Comparison of means and standard deviations of (a) Tape and email, (b) Pour and email as well as (c) Turn and email concerning the different UX aspects.

\subsection{Usage in Multi-User Scenarios and Final Feedback}

After participants completed all tasks and questionnaires, we asked some questions concerning the usage of our merging interaction techniques in collaborative multi-user scenarios and invited the participants to give final feedback. Overall, they mainly stated that the proposed merging interaction techniques will be useful in multi-user settings because they stimulate to talk to each other. One participant said, "I think it was a lot of fun [using the merging interaction techniques] and it is much more collaborative and easier than email. Furthermore, [the merging interaction techniques] are connecting." . Furthermore, another participant said that the interaction techniques would be "a relief in contrast to other interactions" and people would be less distracted compared to emails. For Tape, the main comments comprised that this interaction technique is very easy and fast, especially compared to sending an email. However, participants wondered how this interaction technique will be performed in a larger group: "I think [Tape] can be confusing with many people in terms of space problems [when devices need to be placed next to each other].". Although, participants were not sure how this will work, they think Tape is useful in social situations when you can talk to each other, e. g. to coordinate the merging. Pour received a lot of positive comments, e. g. "[Pour] was fun." and "It feels very intuitive.". 
Since it is a "playful" interaction technique, two participants said the interaction technique would "spice up collaborating". However, it is necessary to avoid accidental pouring or dropping the phone while pouring. The excluding interaction Turn was mentioned least and comments ranged from "can be seen as unfriendly" to "useful in terms of giving feedback". Participants suggested additional oral communication with other people within the group when performing the Turn interaction. Some participants had concerns touching another persons smartphone, e. g. for hygienic, data protection, or private property reasons. They stated that it is important to trust each other and to talk to each other while using the merging interaction techniques. Furthermore, one participant said that it would be useful to have an undo option in case content was sent accidental.

Some participants mentioned that they especially liked that the merging interaction techniques were performed within one mobile application in contrast to sending an email. For email, the participants had to switch to the mail application and back to our study application. An in-app solution seems to increase the assessment in general. As a side effect, during the study conduct we observed that sending email took some time because of bad Wi-Fi connection. Because the proposed interaction techniques are built on the MilkyWay toolbox by Korzetz et al. [10] that provides a Wi-Fi direct connection between the applied devices, the data exchange was very fast.

\section{Findings and Recommendations}

From the results, we derived suggestions for improving the tested merging interaction techniques as well as some further ideas and impressions for merging content in mixed-focus collaboration. The focus of the user study was to investigate our proposed merging interaction techniques regarding their task load and user experience and compare them with a common alternative.

First of all, the user study showed that our proposed merging interaction techniques are suitable for collocated merging scenarios because they are fast to perform, easy to learn and to use. Furthermore, participants stated that these interaction techniques will affect communication and social interaction positively. To improve task completion times of Tape and Pour and increase the rating of dependability, the implementation of the interactions should be revised. As mentioned by some participants, a visual marking would be beneficial for Tape to know where exactly to swipe. We will improve this in further revisions. For Pour additional alternatives of performing the interaction would be beneficial because participants varied the way of pouring the content from one device to another one. For example, some tried to "shake" the content out of one device, which impeded the detection of the interaction. At the same time, it is important that the interactions remain explicit to avoid accidental detection. This leads us to the necessity to reconsider the learning phase because we observed that participants sometimes took a while to perform an interaction technique correctly. Additionally, we interpret the higher values of standard deviation concerning the task completion time that sometimes the interaction techniques worked immedi- 
ately and sometimes after several trials. We applied a learning-by-demonstration approach by the study leader to present the interaction techniques. From our observations we found that participants sometimes still had difficulties with execution speed (Tape), tilt angle (Pour), exact steps (email), and positioning (Pour). We hypothesise that improved feedback mechanisms during performing the interactions would lead to better results and less errors. As a starting point, we will apply visual feedback when the device recognized, for example, a movement but without detecting the concrete interaction technique. With corresponding help functions, we aim at supporting the user.

\subsection{Limitations and Future Work}

In this work, we compared the merging interaction techniques to common email interaction. Participants also mentioned quite often that they would also use messaging services, e. g. WhatsApp. Such services normally compress data, which is one reason why we used email instead. However, a comparison between our proposed interaction techniques and messaging services would be interesting, too, and could substantiate our findings. Nevertheless, for messaging services a good Wi-Fi connection is also necessary. Our merging interactions do not need an external Wi-Fi connection due to the usage of the smartphone sensors.

Whereas we could proof that our proposed merging interaction techniques are usable, attractive, and overall stimulating, we did not investigate in depth how they would be evaluated in multi-user settings. Consequently, applying them in a mixed-focus collaboration scenario would be beneficial to consolidate our findings. However, from the user feedback and the tailoring to merging activities [13], we hypothesise that the merging interaction techniques will be useful in mixed-focus collaboration. Hence, we plan a combined user study that investigates several mobile device-based interaction techniques in collaboration scenarios to receive extensive user feedback.

\section{Conclusion}

In our work, we presented and investigated three merging interaction techniques for mobile devices, namely Tape, Pour, and Turn. The interaction techniques' main aim is to provide an easy and fast way to merge content either bidirectional, unidirectional, or not at all. We developed an Android prototype that was utilized in order to evaluate task load and UX aspects within a user study. We collected data from time measurements, standardized questionnaires (NASATLX and UEQ), and observations. Furthermore, we compared our interactions with a common way to share content, i. e. sending email with attached content.

The results show that especially Tape and Pour performed well in terms of their attractiveness and hedonic quality. Furthermore, the results concerning the task load prove a positive effect. Compared to sending an email, all three mobile device-based interactions for merging performed better. With the collected 
feedback, we will revise our merging interactions and apply them to collaborative multi-user scenarios. With our approach and investigation, we contribute to the field of collaboration technology in terms of mobile device-based interaction techniques for mixed-focus collaboration and provide easy to use interaction techniques for collocated merging.

\section{Acknowledgements}

The European Social Fund (ESF) and the German Federal State of Saxony have funded this work within the project CyPhyMan (100268299). This work is also funded by the German Research Foundation (DFG, Deutsche Forschungsgemeinschaft) as part of Germany's Excellence Strategy - EXC 2050/1 - Project ID 390696704 - Cluster of Excellence "Centre for Tactile Internet with Human-inthe-Loop" (CeTI) of Technische Universität Dresden.

\section{References}

1. Abowd, G.D., Iftode, L., Mitchell, H.: The Smart Phone: A First Platform for Pervasive Computing. IEEE Pervasive Computing 4(2), 18-19 (2005). https://doi.org/10.1016/j.bjps.2009.11.010

2. Ballagas, R., Borchers, J., Rohs, M., Sheridan, J.G.: The Smart Phone: A Ubiquitous Input Device. IEEE Pervasive Computing 5(1), 70-77 (jan 2006). https://doi.org/10.1109/MPRV.2006.18

3. Boring, S., Altendorfer, M., Broll, G., Hilliges, O., Butz, A.: Shoot \& copy: Phonecam-based information transfer from public displays onto mobile phones. In: Proceedings of the 4th International Conference on Mobile Technology, Applications, and Systems and the 1st International Symposium on Computer Human Interaction in Mobile Technology. p. 24-31. Mobility '07, Association for Computing Machinery, New York, NY, USA (2007). https://doi.org/10.1145/1378063.1378068

4. Diehl, M., Stroebe, W.: Productivity loss in brainstorming groups: Toward the solution of a riddle. Journal of Personality and Social Psychology 53, 497-509 (09 1987). https://doi.org/10.1037/0022-3514.53.3.497

5. Dong, T., Churchill, E.F., Nichols, J.: Understanding the Challenges of Designing and Developing Multi-Device Experiences. In: DIS '16: Proceedings of the 2016 ACM Conference on Designing Interactive Systems. pp. 62-72 (2016)

6. Gutwin, C., Greenberg, S.: Design for Individuals, Design for Groups: Tradeoffs Between Power and Workspace Awareness. In: Proceedings of the 1998 ACM conference on Computer supported cooperative work (CSCW'98). pp. 207-216 (1998). https://doi.org/10.1145/289444.289495

7. Hinckley, K.: Synchronous gestures for multiple persons and computers. In: Proceedings of the 16th Annual ACM Symposium on User Interface Software and Technology. p. 149-158. UIST '03, Association for Computing Machinery, New York, NY, USA (2003). https://doi.org/10.1145/964696.964713

8. Hinckley, K., Ramos, G., Guimbretiere, F., Baudisch, P., Smith, M.: Stitching: Pen gestures that span multiple displays. In: Proceedings of the Working Conference on Advanced Visual Interfaces. p. 23-31. AVI '04, Association for Computing Machinery, New York, NY, USA (2004). https://doi.org/10.1145/989863.989866 
9. Korzetz, M., Kühn, R., Heisig, P., Schlegel, T.: Natural Collocated Interactions for Merging Results with Mobile Devices. In: MobileHCI '16: Proceedings of the 18th International Conference on Human-Computer Interaction with Mobile Devices and Services. pp. 746-752 (2016). https://doi.org/10.1145/2957265.2961839

10. Korzetz, M., Kühn, R., Kegel, K., Georgi, L., Schumann, F.W., Schlegel, T.: Milkyway: A toolbox for prototyping collaborative mobile-based interaction techniques. In: Antona, M., Stephanidis, C. (eds.) HCII '19: Universal Access in HumanComputer Interaction. Multimodality and Assistive Environments. pp. 477-490. Springer International Publishing, Cham (2019)

11. Korzetz, M., Kühn, R., Schlegel, T.: Turn it, pour it, twist it: A model for designing mobile device-based interactions. In: CHIuXiD '19: Proceedings of the 5th International ACM In-Cooperation HCI and UX Conference. pp. 20-23. CHIuXiD'19, ACM, New York, NY, USA (2019). https://doi.org/10.1145/3328243.3328246

12. Kühn, R., Korzetz, M., Büschel, L., Korger, C., Manja, P., Schlegel, T.: Natural Voting Interactions for Collaborative Work with Mobile Devices. In: CHI '16: Proceedings of the 34th of the International Conference Extended Abstracts on Human Factors in Computing Systems. pp. 2570-2575 (2016). https://doi.org/10.1145/2851581.2892300

13. Kühn, R., Schlegel, T.: Mixed-Focus Collaboration Activities for Designing Mobile Interactions. In: MobileHCI '18: Proceedings of the 20th International Conference on Human-Computer Interaction with Mobile Devices and Services. pp. 71-78 (2018). https://doi.org/10.1145/3236112.3236122

14. Laugwitz, B., Held, T., Schrepp, M.: Construction and Evaluation of a User Experience Questionnaire. In: HCI and Usability for Education and Work. pp. 63-76 (2008)

15. Leigh, S.w., Schoessler, P., Heibeck, F., Maes, P., Ishii, H.: THAW: Tangible Interaction with See-Through Augmentation for Smartphones on Computer Screens. In: Proc. of the 9th International Conference on Tangible, Embedded, and Embodied Interaction - TEI '15. pp. 89-96 (2015)

16. Lim, H., Ahn, H., Kang, J., Suh, B., Lee, J.: Ubi-jector: An Information-Sharing Workspace in Casual Places Using Mobile Devices. In: Proc. of the 16th international conference on Human-computer interaction with mobile devices \& services (MobileHCI '14). pp. 379-388 (2014)

17. Lucero, A., Jones, M., Jokela, T., Robinson, S.: Mobile collocated interactions: taking an offline break together. Interactions 20(2), 26-32 (2013)

18. Lucero, A., Keränen, J., Jokela, T.: Social and Spatial Interactions: Shared CoLocated Mobile Phone Use. In: Proc. of the 28th of the international conference extended abstracts on Human factors in Computing Systems. pp. 3223-3228. CHI EA '10, ACM Press (2010)

19. Ohta, T.: Intuitive gesture set on touch screen for ad-hoc file sharing among multiple mobile devices. In: 2014 IEEE 3rd Global Conference on Consumer Electronics (GCCE). pp. 751-752 (2014). https://doi.org/10.1109/GCCE.2014.7031188

20. Ohta, T., Tanaka, J.: Movietile: Interactively adjustable free shape multi-display of mobile devices. In: SIGGRAPH Asia 2015 Mobile Graphics and Interactive Applications. SA '15, Association for Computing Machinery, New York, NY, USA (2015). https://doi.org/10.1145/2818427.2818436

21. Paay, J., Raptis, D., Kjeldskov, J., Lauridsen, B.M., Penchev, I.S., Ringhauge, E., Ruder, E.V.: A comparison of techniques for cross-device interaction from mobile devices to large displays. In: Proceedings of the 14th International Conference on Advances in Mobile Computing and Multi Media. p. 137-146. MoMM '16, ACM (2016). https://doi.org/10.1145/3007120.3007140 
22. Rekimoto, J.: Pick-and-drop: A direct manipulation technique for multiple computer environments. In: Proceedings of the 10th Annual ACM Symposium on User Interface Software and Technology. p. 31-39. UIST '97, Association for Computing Machinery, New York, NY, USA (1997). https://doi.org/10.1145/263407.263505

23. Rico, J., Brewster, S.: Usable Gestures for Mobile Interfaces: Evaluating Social Acceptability. In: Proc. of the 28th International Conference on Human Factors in Computing Systems - CHI '10. pp. 887-896 (2010)

24. Seifert, J., Simeone, A., Schmidt, D., Holleis, P., Reinartz, C., Wagner, M., Gellersen, H., Rukzio, E.: MobiSurf: Improving Co-located Collaboration through Integrating Mobile Devices and Interactive Surfaces. In: Proc. of the 2012 ACM international conference on Interactive tabletops and surfaces - ITS '12. pp. 51-60. ACM Press (2012) 